\title{
Comparison of in-hospital and longer-term outcomes of hybrid and Norwood stage 1 palliation of hypoplastic left heart syndrome
}

\author{
Sadia Malik, MBBS, MPH, ${ }^{\mathrm{a}}$ T. Mac Bird, PhD, ${ }^{\mathrm{b}}$ Robert D. B. Jaquiss, MD, ${ }^{\mathrm{c}}$ W. Robert Morrow, MD, ${ }^{\mathrm{d}}$ and \\ James M. Robbins, $\mathrm{PhD}^{\mathrm{a}}$
}

\section{ABSTRACT}

Objectives: The hybrid approach for the initial management of hypoplastic left heart syndrome shifts the risks of major open surgery from the vulnerable neonatal period to an older age. This study determined differences between the hybrid and the standard Norwood procedures in postoperative in-hospital mortality, renal failure, and survival to at least 2 years of age.

Methods: Data from the Pediatric Health Information System, a detailed hospital discharge database of 43 freestanding children's hospitals, were analyzed. The Pediatric Health Information System includes demographic information, diagnosis, and procedure and clinical service data. Instrumental variable regression techniques were used to estimate the predicted probability of in-hospital mortality, renal failure, and survival to 24 months of age for infants with hypoplastic left heart syndrome who received a hybrid or Norwood procedure. The statistical models controlled for demographics and comorbid chromosomal anomalies.

Results: A total of 3654 infants with hypoplastic left heart syndrome underwent intervention from 1998 to 2012. Of these, 242 underwent the hybrid approach and the remainder underwent the Norwood procedure. Instrumental variable models showed significantly reduced odds of patients who underwent the hybrid approach being diagnosed with renal failure (adjusted risk ratio [ARR], 0.48; 95\% confidence interval [CI], 0.26-0.89); increased odds of surviving initial hospitalization (ARR, 1.28; 95\% CI, 1.06-1.55); increased odds of survival, indicated by readmissions more than 6 months after initial hospitalization (ARR, 1.53; 95\% CI, 1.05-2.22); and a decrease in length of stay by 20 days for the initial surgical hospitalization $(95 \% \mathrm{CI},-27.4$ to -13.9$)$.

Conclusions: The short term hospital-based outcomes and longer-term survival outcomes of the hybrid approach for hypoplastic left heart syndrome may be better than those of the Norwood procedure. (J Thorac Cardiovasc Surg 2015;150:474-80)

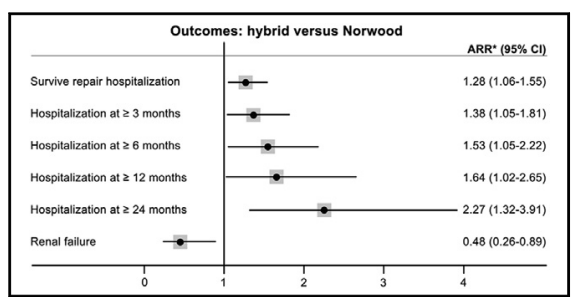

Survival outcomes for stage 1 repair of HLHS may be improved by the hybrid approach compared with the Norwood. *ARR greater than 1 indicates outcome more likely for hybrid.

\section{Central Message}

Survival outcomes for stage 1 repair of HLHS may be improved by the hybrid approach compared with the Norwood.

\section{Perspective}

The Norwood is the traditional initial surgery for HLHS. The alternative hybrid approach shifts risks from the neonatal period to an older age. IV methods were used to adjust for bias in differential selection of 1 treatment option for the most fragile neonates. Survival outcomes were found to be improved for the hybrid compared with the Norwood.

See Editorials pages 456 and 460.
From the a Department of Pediatrics, University of Arkansas for Medical Sciences, Little Rock, Ark; ' ${ }^{\mathrm{b}}$ Department of Health Policy and Management, University of Arkansas for Medical Sciences, Little Rock, Ark; ${ }^{\mathrm{c} D e p a r t m e n t ~ o f ~ C a r d i o t h o r a c i c ~}$ Surgery, Duke University School of Medicine, Durham, NC; and ${ }^{\mathrm{d} C h i l d r e n ' s ~ M e d-~}$ ical Center, Dallas, Tex.

This research was supported in part by Children's University Medical Group research fund intramural grant, Arkansas Children's Hospital Research Institute, Little Rock, Arkansas, and in part by the University of Arkansas for Medical Sciences Translational Research Institute, Grant UL1RR029884 through the National Institutes of Health National Center for Research Resources and National Center for Advancing Translational Sciences. The content is solely the responsibility of the authors and does not necessarily represent the official views of the National Institutes of Health.

Received for publication Sept 22, 2014; revisions received June 8, 2015; accepted for publication June 28, 2015; available ahead of print Aug 1, 2015.

Address for reprints: James M. Robbins, PhD, Department of Pediatrics, University of Arkansas for Medical Sciences, Arkansas Children's Hospital, 1 Children's Way, Little Rock, AR 72202) (E-mail: robbinsjamesm@uams.edu).

0022-5223/\$36.00

Copyright (c) 2015 by The American Association for Thoracic Surgery

http://dx.doi.org/10.1016/j.jtcvs.2015.06.071
丹 Supplemental material is available online.

Congenital heart defects represent approximately $20 \%$ of all major structural birth defects. Hypoplastic left heart syndrome (HLHS) comprises $8 \%$ of congenital heart defects and is associated with significant morbidity and mortality. HLHS accounts for $23 \%$ of cardiac deaths during the first week of life and $15 \%$ of cardiac deaths within the first month of life.

The Norwood procedure has been the traditional initial surgery for HLHS. ${ }^{2}$ Mortality associated with the Norwood procedure at high-volume centers is between $15 \%$ and $17 \%$ despite technical modifications and general improvements in the care of neonates undergoing open surgery. ${ }^{3-5}$ 


\section{Abbreviations and Acronyms \\ $\mathrm{ARR}=$ adjusted risk ratio \\ CI = confidence interval \\ HLHS $=$ hypoplastic left heart syndrome \\ ICD-9 = International Classification of Diseases, Ninth Revision \\ IV = instrumental variable \\ PHIS $=$ Pediatric Health Information System}

During the past 20 years, the hybrid approach has been developed as an alternative for the initial palliation of HLHS. ${ }^{5}$ The hybrid is less surgically invasive than the Norwood in that it does not require cardioplegia or cardiopulmonary bypass during the neonatal period. In a recent analysis of the experience at 100 centers with 1728 neonates receiving stage 1 palliation of HLHS, neonates undergoing the hybrid procedure were found to be at higher presurgical risk than those undergoing the Norwood procedure. ${ }^{6}$ Unadjusted in-hospital mortality was higher for the hybrid (30\%) than the Norwood $(16 \%, P<.001)$ procedure. Karamlou and colleagues, ${ }^{6}$ along with others, ${ }^{7,8}$ have called for studies comparing outcomes of the hybrid and Norwood procedures adjusting for differences in patient risk, and studies comparing outcomes of stage 1 palliation beyond the newborn period.

The present study uses a large multicenter database and the instrumental variable (IV) statistical technique to compare hospital-based, short-term outcomes and survival to at least age 2 years among neonates undergoing the hybrid approach with neonates receiving the traditional Norwood procedure. Quasi-experimental designs such as IV analysis can take advantage of geographic and temporal variation in practice patterns to estimate causal treatment effects by controlling for omitted variables, such as differences in patient risk that influence the choice of one treatment over another. To our knowledge, this is the first study to compare the risk-adjusted outcomes of these 2 approaches and survival outcomes beyond the surgical hospitalization.

\section{MATERIALS AND METHODS}

\section{Data Source}

Data for this study were abstracted from the Pediatric Health Information System (PHIS), an administrative database that contains inpatient data from 43 freestanding children's hospitals in North America (www.chca. com/owner_hospitals/index.html). Participating hospitals are affiliated with the Children's Hospital Association. Data quality and reliability are ensured through a joint effort between the Children's Hospital Association and participating hospitals. For the purposes of external benchmarking, participating hospitals provide discharge data including demographic information and diagnoses and procedures coded with International Classification of Diseases, Ninth Revision (ICD-9) codes. Data are de-identified at the time of data submission and subjected to a number of reliability and validity checks before being processed into data-quality reports. Data are accepted into the database once classified errors occur less frequently than a criterion threshold. Questionable data can be resubmitted and reevaluated before inclusion in the database.

\section{Identification of Study Population}

The study group was defined as all patients with a diagnosis of HLHS (ICD-9 diagnosis code 746.7) who were admitted within the first week of life to a PHIS hospital after January 1, 1998, and discharged before December 31, 2012. Infants with a birth weight less than $1500 \mathrm{~g}$ or more than $4500 \mathrm{~g}$ were excluded to reduce the known risk of morbidity and mortality independent of procedure. Infants were coded as those who underwent the Norwood procedure or the hybrid approach. There was no specific ICD-9-CM procedure code to indicate a Norwood procedure or a hybrid approach. Codes for extracorporeal circulation required for open surgery (ICD-9 procedure code 39.61) and either creation of conduit between right ventricle and pulmonary artery (ICD-9 procedure code 35.92 ) or systemic to pulmonary artery shunt (ICD-9 procedure code 39.0 ) in a patient with the diagnosis of HLHS were used as an indication that the Norwood procedure was performed. The stenting of ductus arteriosus (ICD-9 procedure code 00.45 or ICD-9 procedure code 39.90 ) without extracorporeal circulation (ICD-9 procedure code 39.61 ) in a patient with the diagnosis of HLHS was used as an indication that the hybrid approach was performed. Using this strategy with de-identified patients in our center accurately identified all patients undergoing the hybrid procedure and the Norwood procedure.

\section{Statistical Analysis}

Observational studies of healthcare outcomes are often complicated by nonrandom assignment of treatment based on clinical indication or perceived benefit. These factors driving selection of patients into one form of care or another are often not adequately captured in administrative or registry datasets. For example, most administrative datasets and many registries do not include results of laboratory tests that might influence both a physician's treatment choice and the outcome of that treatment. Not including these laboratory values in the analysis could significantly bias the results, the direction and magnitude of which cannot be predicted analytically. Quasi-experimental designs such as IV analysis offer a technique to control for omitted variable bias by taking advantage of natural experiments that present themselves in the data. IVs can be used to estimate causal treatment effects with observational data if the proper conditions are met. $^{9}$

IV analysis corrects for omitted variable bias in observational studies by identifying a maker, termed an instrument, for a natural experiment. For an instrument to be valid, it must meet 3 key assumptions. The first assumption is that the instrument is associated with treatment assignment, the stronger the association the better. The second assumption is that the instrument is not correlated with unobserved variables that influence the outcome. The third assumption is that the instrument does not directly affect the outcome. The instrument can only affect the outcome through its association with treatment assignment. The first 2 assumptions can be tested empirically, but the third cannot. It must be evaluated by theory. Although the third assumption can be tested by estimating the association of the instrument with the outcome, because of the strong association required between the instrument and the treatment assignment, a statistically significant result does not invalidate the instrument. By meeting these 3 assumptions, an instrument can be used to purge the treatment variable of its correlation with the important omitted variables. This is done by using the instrument to predict treatment assignment. The predicted treatment assignment takes the place of the actual treatment assignment in statistical analysis to produce an unbiased and consistent estimate of the treatment effect. ${ }^{10}$

The IV used in the current study is variation in physician practice patterns at tertiary care children's hospitals. The instrument is a continuous variable constructed by calculating the hospital specific rate, by year, at which patients with HLHS underwent the hybrid procedure as opposed to the Norwood procedure. This instrument is highly correlated with 
treatment assignment, thus meeting the first assumption of a valid instrument. If patient mix and level of care do not differ systematically across major tertiary care children's hospitals, then this instrument should meet the second assumption of a valid instrument. If the instrument, rate of hybrid surgical repair by hospital by year, only affects the outcomes through its association with treatment assignment, then this instrument meets the third assumption of a valid instrument. Variation in physician practice patterns at all levels, from the individual physician to regions of the country has been used as an IV in a number of health services and outcomes studies $^{9}$ and has been validated in Monte Carlo simulation studies. ${ }^{11}$

Control variables for all analyses included birth weight, gender, race/ ethnicity, insurance status, presence of a major comorbid birth defect by body system category, presence of any minor birth defect, number of comorbid heart defects, number of comorbid nonheart defects, birth weight, and an indicator for admission to the tertiary care hospital on the first day of life, hospital HLHS volume, hospital Norwood procedure volume, hospital hybrid procedure volume, and year of hospital admission.

Outcome variables included in-hospital mortality, renal failure, any grade intraventricular hemorrhage, and length of hospital stay. An additional set of outcome variables were constructed as proxies for survival after the initial surgical hospitalization. For each patient, an indicator variable was constructed to represent any readmission to the original surgical hospital at or beyond 3, 6, 12, and 24 months of age. Although the absence of a readmission does not necessarily indicate mortality, a patient could move or otherwise change hospitals, the presence of a readmission is positive proof of survival to that particular age.

For the analyses of dichotomous outcomes, multivariable probit models were estimated. Results for dichotomous outcomes were reported in the form of risk ratios and $95 \%$ confidence intervals (CIs). For analyses of length of hospital stay, the dependent variable was treated as continuous and highly right skewed. For these analyses, a generalized linear model with a gamma distribution and a log link was used. For IV models in which the dependent variable is dichotomous, because the treatment variable also was dichotomous, a bivariate probit model with IVs was used. For the IV analysis of the length of stay outcomes, the 2-stage residual inclusion method was used. ${ }^{12}$ The first stage consists of a multivariable probit model predicting treatment choice, and the second stage consists of a multivariable generalized linear model with a gamma distribution and a log link. IV diagnostic tests were also conducted. Stata MP v.12 statistical software (StataCorp LP, College Station, Tex) was used for all analyses.

\section{RESULTS}

A total of 3654 children with HLHS from 39 hospitals were included in the analyses. Four PHIS hospitals were excluded because they lacked eligible participants during the time frame they submitted data to PHIS. Of all children, 3412 (93.4\%) underwent the Norwood procedure and 242 $(6.6 \%)$ underwent the hybrid approach. Eight hospitals performed no hybrid procedures over the course of the study. Between 1 and 10 hybrid procedures were performed at 26 hospitals, between 11 and 20 hybrid procedures were performed at 3 hospitals, and 30 or more hybrid procedures were performed at 2 hospitals.

Patient characteristics are summarized in Table 1 . There were more white infants in the hybrid group than in the Norwood group ( $61 \%$ vs $52 \%, P=.011)$, and more Hispanic infants in the Norwood group than in the hybrid group $(17 \%$ vs $9 \%, P=.001)$. Patients who received the hybrid procedure had more comorbid central nervous system defects $(2.1 \%$ vs $0.7 \%, P=.021)$, more comorbid orofacial defects $(2.1 \%$ vs $0.7 \%, P=.016)$, and more major comorbid birth defects overall ( 2.5 vs $2.2, P=.028$ ). Patients in the hybrid group also had lower birth weight in grams (3044 vs $3121, P=.037$ ) than patients who received the Norwood approach. Relatively more hybrid procedures were performed in more recent years (2007-2012) than in earlier years. Consistent with the Risk Adjustment for Congenital Heart Surgery scoring of surgical complexity, the mean score for the Norwood was 5.0 compared with 2.1 for the hybrid. The median days of age of procedure was 5 (interquartile range, 2-8) for the hybrid and 4 (interquartile range, 3-7) for the Norwood.

Results of bivariate analyses of all outcomes are presented in Table 2. Infants receiving the hybrid procedure were significantly less likely to be readmitted to the surgical hospital after 3 months (RR, 0.86; 95\% CI, 0.75-0.97), 6 months (RR, 0.72; 95\% CI, 0.60-0.87), 12 months (RR, 0.57 ; $95 \%$ CI, 0.45-0.74), and 24 months (RR, $0.58 ; 95 \%$ CI, 0.44-0.77).

Results of multivariable analyses and IV analyses of dichotomous outcomes are presented in Table 3. Multivariable probit models indicate no statistically significant differences between procedures on any outcome measured except a 10-day $(95 \% \mathrm{CI},-14.8$ to -6.1$)$ shorter length of stay for infants undergoing the hybrid procedure. The IV analyses indicate that patients undergoing the hybrid procedure were significantly more likely to survive the initial surgical hospitalization (adjusted risk ratio [ARR], $1.28 ; 95 \%$ CI, 1.06-1.55); more likely to be readmitted to the surgical hospital after 3 months (ARR, 1.38 ; 95\% CI, 1.05-1.81), 6 months (ARR, 1.53; 95\% CI, 1.05-2.22), 12 months (ARR, 1.64; 95\% CI, 1.02-2.65), and 24 months (ARR, 2.27; 95\% CI, 1.32-3.91); and less likely to be diagnosed with renal failure (ARR, 0.48; 95\% CI, 0.26-0.89). The IV analysis also indicated a 20 -day $(95 \%$ CI, -27.4 to -13.9 ) reduction in length of stay for infants undergoing the hybrid procedure.

A number of sensitivity analyses were conducted. In the first sensitivity analysis, the study was replicated with data beginning in 2006, when hybrid procedures were becoming more widely used. The results were consistent with and in the same direction as those based on the full sample. Loss of patients $(20 \%$ of hybrid cases and $42 \%$ of Norwood cases) with this truncated sample decreased the analytic power of the study, and thus fewer outcomes were statistically significant. Survival of the initial hospitalization (ARR, 1.35 ; $95 \% \mathrm{CI}, 1.04-1.75)$ and survival to hospitalization at or beyond 24 months of age (ARR, $1.80 ; 95 \% \mathrm{CI}$, 1.10-2.92) remained statistically significant, favoring the hybrid. Other sensitivity analyses included restricting the sample to hospitals using the hybrid procedure at least once over the study period, using a dichotomized version of the study's original continuous IV, and using an 
TABLE 1. Distribution of control variables for infants with hypoplastic left heart syndrome undergoing standard Norwood or hybrid repair

\begin{tabular}{|c|c|c|c|}
\hline \multirow[b]{2}{*}{ Control variables } & \multicolumn{3}{|c|}{ Treatment distribution } \\
\hline & Norwood & Hybrid & $P$ value \\
\hline Total n (\%) & $3412(93.4)$ & $242(6.6)$ & - \\
\hline \multicolumn{4}{|l|}{ Demographics } \\
\hline White & 52.3 & 60.7 & .011 \\
\hline Black & 11.4 & 9.9 & .481 \\
\hline Hispanic & 16.7 & 8.7 & .001 \\
\hline Other race & 13.2 & 15.7 & .267 \\
\hline Missing race & 6.6 & 5.4 & .466 \\
\hline Male & 61.9 & 64.5 & .427 \\
\hline Privately insured & 36.1 & 33.9 & .498 \\
\hline Publicly insured & 46.0 & 54.1 & .014 \\
\hline Uninsured & 13.9 & 9.1 & .035 \\
\hline Other insurance & 4.0 & 2.9 & .375 \\
\hline \multicolumn{4}{|l|}{ Major comorbid birth defects } \\
\hline Cardiovascular & 71.0 & 76.5 & .069 \\
\hline Central nervous system & 0.7 & 2.1 & .021 \\
\hline Eye/ear & 0.2 & 0.4 & .322 \\
\hline Orofacial & 0.7 & 2.1 & .016 \\
\hline Gastrointestinal & 1.1 & 1.2 & .892 \\
\hline Genitourinary & 4.4 & 6.2 & .192 \\
\hline Musculoskeletal & 0.5 & 0.0 & .286 \\
\hline Chromosomal & 2.9 & 2.9 & .973 \\
\hline Any minor birth defect & 8.9 & 11.2 & .232 \\
\hline Total major comorbid birth defects & 2.2 & 2.5 & .028 \\
\hline Total major comorbid heart defects & 1.3 & 1.4 & .155 \\
\hline \multicolumn{4}{|l|}{ Year of admission } \\
\hline $1998-2000$ & 8.9 & 0.8 & $<.001$ \\
\hline $2001-2002$ & 11.1 & 3.3 & $<.001$ \\
\hline 2003-2004 & 14.4 & 11.2 & .160 \\
\hline $2005-2006$ & 15.1 & 9.9 & .028 \\
\hline $2007-2008$ & 17.9 & 25.2 & .004 \\
\hline $2009-2010$ & 15.9 & 24.4 & .001 \\
\hline 2011-2012 & 16.7 & 25.2 & .001 \\
\hline Admitted on first day of life & 75.3 & 75.2 & .968 \\
\hline $\begin{array}{l}\text { Hospitalization day of surgical } \\
\text { procedure (mean) }\end{array}$ & 5.3 & 7.7 & $<.001$ \\
\hline Mean birth weight in grams & 3109 & 3023 & .018 \\
\hline Mean RACHS score & 5.0 & 2.1 & $<.001$ \\
\hline
\end{tabular}

alternative IV in which the procedure used to treat the previous patients with HLHS at a particular hospital was used to predict the current patient's treatment procedure. Results of these sensitivity analyses were similar to those of the original analysis. All of these sensitivity analyses were replicated using a generalized methods of moments model instead of the original maximum likelihood model. All results were in the same direction but often no longer statistically significant given that generalized method of moments is generally less efficient than maximum likelihood. The results are shown in Tables E1 to E5.
IV diagnostics were conducted. Compared with the relative imbalance of some control variables seen between treatments in Table 1, control variables were more balanced across dichotomized levels of the instrument (results available from the authors). For each outcome variable, a Hausman test for endogeneity, an F-test for weak instruments, and an F-test for the association with the IV were conducted. Significant endogeneity was detected for all outcomes except renal failure and intraventricular hemorrhage/periventricular leukomalacia. The F-statistic for the test for weak instruments was always greater than 250 , which is well above the rule of thumb cutoff of 10 suggested by Staiger and Stock ${ }^{13}$ typically used to identify a strong instrument. The instrument was associated with 3 of the 8 outcomes: hospital readmission at 12 months or more, renal failure, and length of stay. Diagnostic results are presented in Appendix E1.

\section{DISCUSSION}

This study compares in-hospital outcomes and survival of infants undergoing the hybrid and Norwood procedures across multiple centers. In a recent presentation, Karamlou and colleagues ${ }^{6}$ have shown that infants with higher measurable preoperative risk were more likely to undergo the hybrid procedure than the Norwood. In the current study, traditional multivariable analyses adjusting for measurable preoperative risk indicated similar rates of inhospital mortality, similar rates of renal failure, and similar evidence of increased survival to at least 2 years of age in infants undergoing the hybrid or Norwood.

However, traditional multivariable analysis can only control for differences in risk identified by measured variables. If unmeasured risk factors are in part driving the calculus behind surgical choice, resulting in higher-risk infants more often undergoing the hybrid procedure, results will be biased toward the Norwood. IV analysis is a quasiexperimental analytic method, largely developed and refined in the field of economics, which is capable of controlling for omitted variable bias, such as unmeasured risk factors, so long as key assumptions are met.

The IV methods used in the current study indicated that patients undergoing the hybrid procedure were significantly less likely to experience renal failure, more likely to survive in both the short term and up to 24 months of age, and spent approximately 3 weeks less time in the hospital postsurgery than patients undergoing the Norwood. These results, based on a larger sample than available from single centers, were sufficiently powered to detect a true difference between groups in neonatal outcomes if they existed.

Taken together, divergent findings between multivariate models demonstrating a minimal difference between the hybrid and the Norwood and IV models showing a clear advantage to the hybrid procedure suggests that there is significant and unmeasured selection bias in the surgeon's 
TABLE 2. Bivariate analysis of outcomes for neonates with hypoplastic left heart syndrome undergoing standard Norwood or hybrid repair

\begin{tabular}{lcccc}
\hline \multicolumn{1}{c}{ Clinical outcomes } & Norwood & Hybrid & RR & 95\% CI \\
\cline { 2 - 4 } & rate (\%) & rate (\%) & 0.98 & $0.91-1.05$ \\
\hline Repair hospital survival & 78.3 & 76.9 & $\mathbf{0 . 8 6}$ & $\mathbf{0 . 7 5 - 0 . 9 7}$ \\
Hospitalization $\geq 3$ mo & 60.4 & 51.7 & $\mathbf{0 . 7 2}$ & $\mathbf{0 . 6 0 - 0 . 8 7}$ \\
Hospitalization $\geq 6$ mo & 45.6 & 33.1 & $\mathbf{0 . 5 7}$ & $\mathbf{0 . 4 5 - 0 . 7 4}$ \\
Hospitalization $\geq 12$ mo & 37.3 & 21.5 & 0.73 & $0.48-1.12$ \\
Hospitalization $\geq 24$ mo & 30.5 & 8.7 & 2.17 & $0.49-9.56$ \\
Renal failure & 11.8 & 0.8 & Difference \\
Any IVH or PVL & 0.4 & Mean & 0.24 \\
\hline \multicolumn{1}{c}{ Use outcomes } & Mean & 42.1 & $\mathbf{9 5} \%$ CI \\
\hline Total days in hospital & 41.9 & $-3.95-4.43$ \\
\hline
\end{tabular}

Bold indicates statistical significance at $P<.05$. CI, Confidence interval; $I V H$, intraventricular hemorrhage; $P V L$, periventricular leukomalacia; $R R$, risk ratio.

choice of approach. The subtlety of clinical risk assessment in this complex group of patients is notoriously difficult to quantify, and so the inadequacy of standard multivariate models to fully adjust for clinical risk in this situation is not surprising.

In 1993, Gibbs and colleagues ${ }^{14}$ described the hybrid approach as an alternative to the Norwood procedure for initial palliation of HLHS. The hybrid approach controls pulmonary blood flow and maintains unobstructed systemic cardiac output without the need for open surgery. Open surgery during neonatal life is associated with increased mortality and morbidity. The hybrid approach also is a less-invasive option than the Norwood procedure for HLHS patients waiting for transplantation. ${ }^{15}$ Among patients with HLHS with borderline 2 ventricles, palliation using the hybrid approach provides a chance for further growth or functional recovery of the ventricles and makes 2-ventricle repair possible. ${ }^{16}$

Over the last few years, numerous single-center studies have shown success of the hybrid approach for HLHS in short-term hospital-based outcomes. ${ }^{17}$ In Giessen,
Germany, ${ }^{18} 58$ newborns underwent ductal stenting and bilateral pulmonary artery banding between 1998 and 2006. The 30-day mortality rate for combined ductal stent placement and bilateral pulmonary artery banding was $3.4 \%$. The single-center experience from Columbus Children's Hospital ${ }^{19}$ included 62 patients who underwent the hybrid procedure between 2002 and 2007. This study excluded patients (1) with non-HLHS univentricular anatomy; (2) bridged to a 2-ventricle repair; (3) at known high risk, including intact atrial septum, weight less than $1.5 \mathrm{~kg}$, and significant extracardiac malformations; and (4) who were part of the their initial learning curve. With these exclusions, $97.5 \%$ (39 of 40) survived the initial hospital stay without the need for extracorporeal membrane oxygenation.

There are subpopulations of infants in whom the Norwood procedure is associated with a higher likelihood of perioperative complications or death. Risk factors for these infants include shock at presentation, birth weight less than $2.5 \mathrm{~kg}$, prematurity less than 34 weeks of gestation, age more than 30 days, aortic atresia, poor right ventricular

TABLE 3. Traditional multivariate analysis and instrumental variable analysis of clinical outcomes for neonates with hypoplastic left heart syndrome undergoing standard Norwood or hybrid repair

\begin{tabular}{|c|c|c|c|c|}
\hline \multirow[b]{2}{*}{ Clinical outcomes } & \multicolumn{2}{|c|}{ Multivariable probit models } & \multicolumn{2}{|c|}{ Bivariate probit with IV models } \\
\hline & ARR & $95 \% \mathbf{C I}$ & ARR & $95 \%$ CI \\
\hline Repair hospital survival & 1.00 & $0.92-1.08$ & 1.28 & 1.06-1.55 \\
\hline Hospitalization $\geq 3 \mathrm{mo}$ & 0.97 & $0.85-1.11$ & 1.38 & $1.05-1.81$ \\
\hline Hospitalization $\geq 6 \mathrm{mo}$ & 1.00 & $0.82-1.20$ & 1.53 & $1.05-2.22$ \\
\hline Hospitalization $\geq 12 \mathrm{mo}$ & 0.96 & $0.78-1.18$ & 1.64 & $1.02-2.65$ \\
\hline Hospitalization $\geq 24$ mo & 1.05 & $0.84-1.32$ & 2.27 & 1.32-3.91 \\
\hline Renal failure & 0.65 & $0.41-1.03$ & 0.48 & $0.26-0.89$ \\
\hline \multirow[t]{2}{*}{ Any IVH or PVL } & 0.79 & $0.19-3.23$ & 0.17 & $0.01-2.82$ \\
\hline & \multicolumn{2}{|c|}{ Generalized linear models } & \multicolumn{2}{|c|}{ 2-stage residual inclusion IV model } \\
\hline Use outcome & Coefficient & $\mathbf{9 5} \% \mathbf{C I}$ & Coefficient & $95 \% \mathrm{CI}$ \\
\hline Total days in hospital & -10.4 & -14.8 to -6.1 & -20.6 & -27.4 to -13.9 \\
\hline
\end{tabular}


function, tricuspid regurgitation, intact atrial septum, and the presence of noncardiac malformations. ${ }^{20}$ In neonates with 1 or several of these risk factors, survival is significantly lower, even at high-volume centers. ${ }^{4}$

The hybrid procedure may be the first choice in certain high-risk groups. The University of Virginia described the results of a univentricular palliation protocol based on a risk-stratified selection of patients for a purely surgical reconstruction or hybrid catheter-surgical palliation. ${ }^{21}$ Operative survival for 10 infants with HLHS without high-risk features undergoing a Norwood/Sano procedure was $90 \%$. Operative survival for 5 infants with HLHS with high-risk features undergoing hybrid palliation was $100 \%$, compared with $29 \%$ in 7 infants with high-risk features undergoing the Norwood/Sano procedure. Between 2003 and 2005, 14 high-risk consecutive neonates underwent a hybrid stage I procedure at the University of Chicago Children's Hospital. ${ }^{22}$ No patient was excluded from the study. Hospital survival was $78.5 \%$ (11 of 14). This pilot series demonstrates that acceptable results can be achieved with the hybrid approach in a very high-risk group.

Although many observational studies are well designed with high-quality clinical data, even the highest quality nonrandomized studies can suffer from selection bias if such bias is not specifically modeled and addressed. We estimated the comparative safety and survival up to 24 months of the hybrid versus the Norwood procedure for the first stage repair of HLHS using IV analysis, a quasiexperimental method designed to account for forms of omitted variable bias, such as selection bias. Although the administrative data used in this study do not have the clinical depth of data gathered in many of the single institution studies previously conducted, the limitations of administrative data are the kinds of limitations IV analysis is well suited to address. ${ }^{20}$

\section{Study Limitations}

This study has limitations that should be considered. Only freestanding children's hospitals are included in the PHIS database. Pediatric cardiac surgical units not within children's hospitals are not included. The analysis included multiple centers treating high-risk patients and patients from hospitals with low volumes that may still be developing techniques for the novel hybrid procedure. Changes in the "learning curve" of these hospitals could influence subsequent analyses. PHIS is an administrative database and is limited in the potential inaccuracies of data collected primarily for billing purposes. However, the database does undergo considerable logic and validity checks beyond that of many large administrative databases to ensure data quality and reliability.

Much of the design of the current study is based on diagnosis or procedure codes. Hospital discharge data are known to underreport many clinical conditions and procedures. ${ }^{23-26}$ Hospital discharge data also may be prone to clerical errors, misclassification, systematic trends in coding bias, and the effects of differential reimbursement rates. If a diagnosis or procedure adds little to reimbursement, it is less likely to be coded than a highly reimbursable diagnosis or procedure.

We have used only ICD-9 procedure codes to uniquely identify neonates who have undergone the hybrid or the Norwood procedure. Although we have validated these definitions with clinical data from identified patients at our center, we have not validated the definitions at other centers. Pasquali and colleagues ${ }^{27}$ have shown that use of ICD-9 diagnosis and procedure codes to identify cardiac surgery when compared with surgery identified in the clinical record overestimates mortality for Risk Adjustment for Congenital Heart Surgery-1 category 2 procedures and underestimates mortality for category 5 procedures. Similar bias if present in our analysis would serve to underestimate survival associated with the hybrid (a category 2 procedure) and overestimate survival associated with the Norwood (a category 5 or 6 procedure). Our results favoring the hybrid procedure would be conservative estimates of the mortality advantage we observed.

Because the data were not collected with this specific study in mind, omitted variable bias could be a significant concern. Although the statistical methods used in the current study are designed to address measurement error and omitted variable bias, the assumptions of the IV model must be met for this to occur. Because at least 1 of the IV assumptions must be evaluated purely on theoretic grounds, it is possible that the chosen instruments are not valid, thus producing biased results. However, we do not believe that to be the case in this study.

This study offers only incomplete evidence of long-term survival. In absolute numbers, a greater proportion of infants undergoing the Norwood were located and alive as evidence of readmission to the same hospital at or beyond 3, 6, 12 , and 24 months of age than infants undergoing the hybrid procedure. In contrast, controlled for omitted variable bias, a greater proportion of infants undergoing the hybrid procedure were located and alive in the hospital at 3, 6, 12, and 24 months of age. We have no information about the infants who have not returned to the same hospital, although we have no reason to anticipate that the type of surgical procedure is related to receiving follow-up care at another hospital. Delaying risk to the second operation has shown no difference in mortality between the hybrid and Norwood procedures in a single-center study. ${ }^{21}$ Results from this PHIS cohort at up to 24 months of age would provide a larger, more representative sample of mortality outcomes.

\section{CONCLUSIONS}

On the basis of a large US cohort, the hybrid approach may represent an improvement over the Norwood 
procedure in neonatal and longer-term outcomes. ${ }^{28}$ Future research should confirm the survival advantage of infants undergoing the hybrid procedure and address longer-term neurologic outcomes in the vulnerable neonate undergoing the hybrid procedure.

\section{Conflict of Interest Statement}

Authors have nothing to disclose with regard to commercial support.

The authors thank Michiaki Imamura, MD, for review of early versions of the article.

\section{References}

1. Cleves MA, Ghaffar S, Zhao W, Mosley BS, Hobbs CA. First-year survival of infants born with congenital heart defects in Arkansas (1993-1998): a survival analysis using registry data. Birth Defects Res A Clin Mol Teratol. 2003;67: $662-8$.

2. Norwood WI, Lang P, Casteneda AR, Campbell DN. Experience with operations for hypoplastic left heart syndrome. J Thorac Cardiovasc Surg. 1981;82:511-9.

3. Sano S, Ishino K, Kawada M, Arai S, Kasahara S, Asai T, et al. Right ventriclepulmonary artery shunt in first-stage palliation of hypoplastic left heart syndrome. J Thorac Cardiovasc Surg. 2003;126:504-9.

4. Hirsch JC, Copeland G, Donohue JE, Kirby RS, Grigorescu V, Gurney JG. Population-based analysis of survival for hypoplastic left heart syndrome. J Pediatr. 2011;159:57-63.

5. Venugopal PS, Luna KP, Anderson DR, Austin CB, Rosenthal E, Krasemann T, et al. Hybrid procedure as an alternative to surgical palliation of high-risk infants with hypoplastic left heart syndrome and its variants. J Thorac Cardiovasc Surg. 2010;139:1211-5.

6. Karamlou T, Overman D, Hill KD, Wallace A, Pasquali SK, Jacobs JP, et al. Stage 1 hybrid palliation for hypoplastic left heart syndrome-assessment of contemporary patterns of use: an analysis of the society of thoracic surgeons congenital heart surgery database. J Thorac Cardiovasc Surg. 2015;149:195-201. 202.e1.

7. Caldarone CA. W(h)ither the hybrid? Up the "slope of enlightenment" J Thorac Cardiovasc Surg. 2015;149:7-8.

8. Davies RR. Editorial commentary: stage 1 hybrid palliation for hypoplastic left heart syndrome - assessment of contemporary patterns of use: an analysis of the society of thoracic surgeons congenital heart surgery database. J Thorac Cardiovasc Surg. 2015;149:203-4.

9. Stukel TA, Fisher ES, Wennberg DE, Alter DA, Gottlieb DJ, Vermeulen MJ. Analysis of observational studies in the presence of treatment selection bias: effects of invasive cardiac management on AMI survival using propensity score and instrumental variable methods. JAMA. 2007;297:278-85.

10. Rassen JA, Brookhart MA, Glynn RJ, Mittleman MA, Schneeweiss S. Instrumental variables I: instrumental variables exploit natural variation in nonexperimental data to estimate causal relationships. J Clin Epidemiol. 2009; 62:1226-32.

11. Rassen JA, Brookhart MA, Glynn RJ, Mittleman MA, Schneeweiss S. Instrumental variables II: Instrumental variable application-in 25 variations, the physician prescribing preference generally was strong and reduced covariate imbalance. J Clin Epidemiol. 2009;62:1233-41.
12. Terza JV, Basu A, Rathouz PJ. Two-stage residual inclusion estimation: addressing endogeneity in health econometric modeling. J Health Econ. 2008;27: $531-43$.

13. Staiger DO, Stock JH. Instrumental variables regression with weak instruments. Econometrica. 1997;65:557-86.

14. Gibbs JL, Wren C, Watterson KG, Hunter S, Hamilton JR. Stenting of the arterial duct combined with banding of the pulmonary arteries and atrial septectomy or septostomy: a new approach to palliation for the hypoplastic left heart syndrome. Br Heart J. 1993;69:551-5.

15. Sebastian VA, Guleserian KJ, Leonard SR, Forbess JM. Heart transplantation techniques after hybrid single-ventricle palliation. J Card Surg. 2010;25: 596-600.

16. Moon-Grady AJ, Moore P, Azakie A. Ross-Konno and endocardial fibroelastosis resection after hybrid stage I palliation in infancy: successful staged leftventricular rehabilitation and conversion to biventricular circulation after fetal diagnosis of aortic stenosis. Pediatr Cardiol. 2011;32:211-4.

17. Pizarro C, Murdison KA, Derby CD, Radtke W. Stage II reconstruction after hybrid palliation for high-risk patients with a single ventricle. Ann Thorac Surg. 2008;85:1382-8.

18. Akintürk H, Michel-Behnke I, Valeske K, Mueller M, Thul J, Bauer J, et al. Hybrid transcatheter-surgical palliation. Pediatr Cardiol. 2007;28:79-87.

19. Galantowicz M, Cheatham JP, Phillips A, Cua CL, Hoffman TM, Hill SL, et al. Hybrid approach for hypoplastic left heart syndrome: intermediate results after the learning curve. Ann Thorac Surg. 2008;85:2063-71.

20. Sarajuuri A, Jokinen E, Puosi R, Eronen M, Mildh L, Mattila I, et al. Neurodevelopmental and neuroradiologic outcomes in patients with univentricular heart aged 5 to 7 years: related risk factor analysis. J Thorac Cardiovasc Surg. 2007; $133: 1524-32$

21. Lim D, Peeler B, Matherne G, Kron I, Gutgesell H. Risk-stratified approach to hybrid transcatheter-surgical palliation of hypoplastic left heart syndrome. Pediatr Cardiol. 2006;27:91-5.

22. Bacha EA, Daves S, Hardin J, Abdulla R, Anderson J, Kahana M, et al. Singleventricle palliation for high-risk neonates: the emergence of an alternative hybrid stage I strategy. J Thorac Cardiovasc Surg. 2006;131:163-71.e2.

23. DiGiuseppe DL, Aron DC, Ranbom L, Harper DL, Rosenthal GE. Reliability of birth certificate data: a multi-hospital comparison to medical records information. Matern Child Health J. 2002;6:169-79.

24. Roohan PJ, Josberger RE, Acar J, Dabir P, Feder HM, Gagliano PJ. Validation of birth certificate data in New York State. J Community Health. 2003;28: 335-46.

25. Korst LM, Gregory KD, Gornbein JA. Elective primary caesarean delivery: accuracy of administrative data. Paediatr Perinat Epidemiol. 2004;18:112-9.

26. Yasmeen S, Romano PS, Schembri ME, Keyzer JM, Gilbert WM. Accuracy of obstetric diagnoses and procedures in hospital discharge data. Obstet Gynecol. 2006;194:992-1001.

27. Pasquali SK, Peterson ED, Jacobs JP, He X, Li JS, Jacobs ML, et al. Differential case ascertainment in clinical registry versus administrative data and impact on outcomes assessment for pediatric cardiac operations. Ann Thorac Surg. 2013; 95:197-203.

28. Galantowicz M. In favor of the hybrid stage 1 as the initial palliation for hypoplastic left heart syndrome. Semin Thorac Cardiovasc Surg Pediatr Card Surg Апnu. 2013;16:62-4.

Key Words: hypoplastic left heart syndrome, Norwood, hybrid, outcomes, instrumental variables 
APPENDIX E1. Instrumental variable diagnostics

\begin{tabular}{|c|c|c|c|c|c|c|}
\hline \multirow[b]{2}{*}{ Clinical outcomes } & \multicolumn{2}{|c|}{ Test for endogeneity } & \multicolumn{2}{|c|}{ Test for weak instruments } & \multicolumn{2}{|c|}{ Association between IV and outcome } \\
\hline & F-statistic & $P$ value & F-statistic & $P$ value & F-statistic & $P$ value \\
\hline Repair hospital survival & 8.2 & .004 & 268.2 & $<.001$ & 0.15 & .702 \\
\hline Hospitalization $\geq 3 \mathrm{mo}$ & 8.0 & .005 & 267.4 & $<.001$ & 1.71 & .191 \\
\hline Hospitalization $\geq 6 \mathrm{mo}$ & 7.2 & .007 & 265.3 & $<.001$ & 2.61 & .107 \\
\hline Hospitalization $\geq 12$ mo & 8.0 & .005 & 261.2 & $<.001$ & 5.81 & .016 \\
\hline Hospitalization $\geq 24$ mo & 10.6 & .001 & 258.3 & $<.001$ & 3.61 & .057 \\
\hline Renal failure & 1.8 & .239 & 265.1 & $<.001$ & 5.61 & .018 \\
\hline \multirow[t]{2}{*}{ Any IVH or PVL } & 0.9 & .334 & 268.0 & $<.001$ & 1.57 & .210 \\
\hline & \multicolumn{2}{|c|}{ Test for endogeneity } & \multicolumn{2}{|c|}{ Test for weak instruments } & \multicolumn{2}{|c|}{ Association between IV and outcome } \\
\hline Use outcome & F-Statistic & $P$ value & F-Statistic & $P$ value & F-Statistic & $P$ value \\
\hline Total days in hospital & 8 & .005 & 281.8 & $<.001$ & 12.04 & .001 \\
\hline
\end{tabular}

$I V$, Instrumental variable; $I V H$, intraventricular hemorrhage; $P V L$, periventricular leukomalacia.

TABLE E1. Maximum likelihood and generalized method of moments analyses, all hospitals, 2000-2012, hospital hybrid rate by year, continuous instrumental variable

\begin{tabular}{|c|c|c|c|c|c|c|c|c|}
\hline & \multicolumn{4}{|c|}{ Maximum likelihood } & \multicolumn{4}{|c|}{ Generalized method of moments } \\
\hline & coefficient* $*$ & $P$ value & $\mathbf{L L}$ & $\mathbf{U L}$ & coefficient $^{*}$ & $P$ value & $\mathbf{L} \mathbf{L}$ & $\mathbf{U L}$ \\
\hline Survive hospitalization & 0.507 & .003 & 0.168 & 0.846 & 0.675 & .012 & 0.150 & 1.200 \\
\hline Survive $3 \mathrm{mo}$ & 0.388 & .012 & 0.084 & 0.692 & 0.441 & .026 & 0.053 & 0.829 \\
\hline Survive $6 \mathrm{mo}$ & 0.371 & .018 & 0.062 & 0.679 & 0.357 & .059 & -0.014 & 0.728 \\
\hline Survive $12 \mathrm{mo}$ & 0.362 & .034 & 0.027 & 0.698 & 0.259 & .181 & -0.121 & 0.639 \\
\hline Survive 24 mo & 0.544 & .002 & 0.197 & 0.892 & 0.381 & .054 & -0.007 & 0.768 \\
\hline Renal failure & -0.466 & .023 & -0.868 & -0.064 & -1.452 & .109 & -3.225 & 0.322 \\
\hline Any IVH or PVL & -0.844 & .226 & -2.210 & 0.523 & \multicolumn{3}{|c|}{ Convergence not achieved. } & \\
\hline Length of stay $\dagger$ & -20.635 & .000 & -27.417 & -13.853 & -27.404 & .000 & -38.109 & -16.700 \\
\hline
\end{tabular}

$I V H$, Intraventricular hemorrhage; $L L$, lower limit of $95 \%$ confidence interval; $P V L$, periventricular leukomalacia; $U L$, upper limit of $95 \%$ confidence interval. *Coefficients $>0$ indicate outcome more likely with hybrid. $\dagger$ Coefficient for length of stay indicates number of fewer days with hybrid.

TABLE E2. Maximum likelihood and generalized method of moments analyses, only hospitals with at least 1 hybrid procedure, 2000-2012, hospital hybrid rate by year, continuous instrumental variable

\begin{tabular}{|c|c|c|c|c|c|c|c|c|}
\hline & \multicolumn{4}{|c|}{ Maximum likelihood } & \multicolumn{4}{|c|}{ Generalized method of moments } \\
\hline & coefficient $^{*}$ & $P$ value & $\mathbf{L L}$ & $\mathbf{U L}$ & coefficient ${ }^{*}$ & $P$ value & $\mathbf{L} \mathbf{L}$ & UL \\
\hline Survive hospitalization & 0.706 & .000 & 0.351 & 1.060 & 0.622 & .020 & 0.098 & 1.147 \\
\hline Survive $3 \mathrm{mo}$ & 0.485 & .002 & 0.176 & 0.793 & 0.435 & .030 & 0.041 & 0.829 \\
\hline Survive 6 mo & 0.435 & .006 & 0.126 & 0.743 & 0.333 & .082 & -0.042 & 0.708 \\
\hline Survive $12 \mathrm{mo}$ & 0.413 & .014 & 0.084 & 0.743 & 0.234 & .232 & -0.150 & 0.619 \\
\hline Survive $24 \mathrm{mo}$ & 0.634 & .000 & 0.304 & 0.963 & 0.388 & .052 & -0.003 & 0.780 \\
\hline Renal failure & -0.513 & .017 & -0.933 & -0.092 & -1.483 & .119 & -3.345 & 0.380 \\
\hline Any IVH or PVL & -0.471 & .447 & -1.684 & 0.742 & \multicolumn{3}{|c|}{ Convergence not achieved. } & \\
\hline Length of stay $\dagger$ & -22.076 & .000 & -29.291 & -14.861 & -24.760 & .000 & -35.513 & -14.008 \\
\hline
\end{tabular}

$I V H$, Intraventricular hemorrhage; $L L$, lower limit of $95 \%$ confidence interval; $P V L$, periventricular leukomalacia; $U L$, upper limit of $95 \%$ confidence interval. *Coefficients $>0$ indicate outcome more likely with hybrid. †Coefficient for length of stay indicates number of fewer days with hybrid. 
TABLE E3. Maximum likelihood and generalized method of moments analyses, all hospitals, 2000-2012, hospital hybrid rate by year, instrumental variable dichotomized at median

\begin{tabular}{|c|c|c|c|c|c|c|c|c|}
\hline & \multicolumn{4}{|c|}{ Maximum likelihood } & \multicolumn{4}{|c|}{ Generalized method of moments } \\
\hline & coefficient* & $P$ value & $\mathbf{L L}$ & $\mathbf{U L}$ & coefficient* & $P$ value & $\mathbf{L L}$ & $\mathbf{U L}$ \\
\hline Survive hospitalization & 0.926 & .000 & 0.482 & 1.369 & 3.979 & .341 & -4.211 & 12.169 \\
\hline Survive 3 mo & 0.535 & .033 & 0.042 & 1.027 & 0.653 & .266 & -0.498 & 1.804 \\
\hline Survive 6 mo & 0.399 & .142 & -0.134 & 0.933 & 0.214 & .681 & -0.807 & 1.235 \\
\hline Survive $12 \mathrm{mo}$ & 0.578 & .140 & -0.190 & 1.347 & -0.465 & .431 & -1.622 & 0.693 \\
\hline Survive 24 mo & 0.700 & .141 & -0.233 & 1.634 & -0.854 & .231 & -2.253 & 0.544 \\
\hline Renal failure & -1.399 & .000 & -1.644 & -1.154 & \multicolumn{3}{|c|}{ Convergence not achieved } & \\
\hline Any IVH or PVL & -0.995 & .034 & -1.916 & -0.074 & \multicolumn{3}{|c|}{ Convergence not achieved } & \\
\hline Length of stay $\dagger$ & -33.694 & .000 & -43.708 & -23.679 & -61.852 & .000 & -93.518 & -30.187 \\
\hline
\end{tabular}

$I V H$, Intraventricular hemorrhage; $L L$, lower limit of $95 \%$ confidence interval; $P V L$, periventricular leukomalacia; $U L$, upper limit of $95 \%$ confidence interval. *Coefficients $>0$ indicate outcome more likely with hybrid. †Coefficient for length of stay indicates number of fewer days with hybrid.

TABLE E4. Maximum likelihood and generalized method of moments analyses, all hospitals, years 2006-2012, hospital hybrid rate by year, continuous instrumental variable

\begin{tabular}{|c|c|c|c|c|c|c|c|c|}
\hline & \multicolumn{4}{|c|}{ Maximum likelihood } & \multicolumn{4}{|c|}{ Generalized method of moments } \\
\hline & coefficient* & $P$ value & $\mathbf{L L}$ & UL & coefficient* & $P$ value & LL & $\mathbf{U L}$ \\
\hline Survive hospitalization & 0.607 & .005 & 0.183 & 1.030 & 0.945 & .010 & 0.229 & 1.662 \\
\hline Survive 3 mo & 0.343 & .061 & -0.015 & 0.701 & 0.458 & .044 & 0.013 & 0.903 \\
\hline Survive 6 mo & 0.305 & .085 & -0.042 & 0.651 & 0.419 & .049 & 0.002 & 0.835 \\
\hline Survive $12 \mathrm{mo}$ & 0.322 & .092 & -0.053 & 0.697 & 0.388 & .076 & -0.041 & 0.817 \\
\hline Survive 24 mo & 0.505 & .011 & 0.118 & 0.892 & 0.496 & .027 & 0.056 & 0.936 \\
\hline Renal failure & -0.531 & .049 & -1.062 & -0.001 & -1.972 & .253 & -5.357 & 1.413 \\
\hline Any IVH or PVL & 0.032 & .958 & -1.183 & 1.247 & -0.578 & .554 & -2.490 & 1.335 \\
\hline Length of stay $\dagger$ & -22.300 & .000 & -31.780 & -12.820 & -30.060 & .000 & -43.264 & -16.856 \\
\hline
\end{tabular}

$I V H$, Intraventricular hemorrhage; $L L$, lower limit of $95 \%$ confidence interval; $P V L$, periventricular leukomalacia; $U L$, upper limit of $95 \%$ confidence interval. *Coefficients > 0 indicate outcome more likely with hybrid. †Coefficient for length of stay indicates number of fewer days with hybrid.

TABLE E5. Maximum likelihood and generalized method of moments analyses, all hospitals, 2000-2012, previous patient procedure instrumental variable

\begin{tabular}{|c|c|c|c|c|c|c|c|c|}
\hline & \multicolumn{4}{|c|}{ Maximum likelihood } & \multicolumn{4}{|c|}{ Generalized method of moments } \\
\hline & coefficient* & $P$ value & $\mathbf{L L}$ & UL & coefficient* & $P$ value & $\mathbf{L L}$ & $\mathbf{U L}$ \\
\hline Survive hospitalization & 0.467 & .035 & 0.032 & 0.902 & 1.146 & .028 & 0.122 & 2.169 \\
\hline Survive 3 mo & 0.462 & .020 & 0.072 & 0.852 & 0.430 & .153 & -0.160 & 1.020 \\
\hline Survive 6 mo & 0.445 & .033 & 0.036 & 0.854 & -0.994 & .000 & -1.380 & -0.608 \\
\hline Survive $12 \mathrm{mo}$ & 0.399 & .099 & -0.075 & 0.873 & -0.234 & .423 & -0.808 & 0.339 \\
\hline Survive 24 mo & 0.646 & .012 & 0.140 & 1.153 & -0.233 & .442 & -0.826 & 0.361 \\
\hline Renal failure & -0.332 & .213 & -0.855 & 0.191 & -0.653 & .222 & -1.702 & 0.395 \\
\hline Any IVH or PVL & -0.693 & .388 & -2.266 & 0.879 & \multicolumn{3}{|c|}{ Convergence not achieved. } & \\
\hline Length of stay $\dagger$ & -24.397 & .000 & -34.573 & -14.221 & -17.198 & .069 & -35.765 & 1.369 \\
\hline
\end{tabular}

